\title{
Sorption de l'atrazine et du diuron sur charbon actif en poudre en présence de tensioactifs, ions calcium et bichromate. Essai de modélisation

Sorption of atrazine and diuron on powdered activated carbon
in the presence of surfactants, calcium and dichromate ions -
Modelling trial

\author{
A. Yaacoubi et J. Ayele
}

Volume 12, numéro 2, 1999

URI : https://id.erudit.org/iderudit/705357ar

DOI : https://doi.org/10.7202/705357ar

Aller au sommaire du numéro

\section{Éditeur(s)}

Université du Québec - INRS-Eau, Terre et Environnement (INRS-ETE)

\section{ISSN}

0992-7158 (imprimé)

1718-8598 (numérique)

\section{Découvrir la revue}

Citer cet article

Yaacoubi, A. \& Ayele, J. (1999). Sorption de l'atrazine et du diuron sur charbon actif en poudre en présence de tensioactifs, ions calcium et bichromate. Essai de modélisation. Revue des sciences de l'eau / Journal of Water Science, 12(2), 389-406. https://doi.org/10.7202/705357ar

\section{Résumé de l'article}

La contamination des eaux naturelles par les micropolluants organiques tels que les herbicides, nécessite le recours à des procédés très performants, en particulier l'adsorption sur charbon actif pour satisfaire aux normes de potabilité sur les eaux distribuées. Si le charbon actif en poudre (CAP) présente une bonne efficacité pour l'élimination des herbicides seuls dans l'eau distillée, la présence dans l'eau d'autres composés organiques et / ou minéraux aura pour conséquence la modification des paramètres de l'équilibre de l'adsorption du système initial et la modification des performances du matériau adsorbant. Cette étude a montré que le CAP utilisé présente une bonne affinité pour les deux herbicides étudiés (atrazine et diuron), dont les capacités d'adsorption, déterminées par le modèle de Langmuir, sont 1,42 et 1,72 mmol.g ${ }^{-1}$ respectivement. La présence de composés organiques tels que les tensioactifs anionique le dodécylsulfate de sodium (DSS), et cationique le bromure d'hexadécyl triméthyl ammonium (BHTA) provoque une forte diminution de la capacité d'adsorption des deux herbicides par le charbon actif. Le BHTA provoque l'effet le plus marqué, par contre, les ions calcium et bichromate n'ont pas d'influence sur l'adsorption des deux herbicides. Les essais de désorption ont montré que l'introduction des coadsorbats organiques provoque la désorption d'une partie de l'herbicide. Ce phénomène est accentué dans le cas du DSS en présence d'ions calcium. L'application de modèles simples de coadsorption, a permis de mettre en évidence l'existence presque exclusive de sites spécifiques d'adsorption pour chacun des substrats étudiés et une inhibition non compétitive due a une interdépendance possible entre les sites d'adsorption et une altération des interactions de chaque composé à la surface du charbon. 


\section{Sorption de l'atrazine et du diuron sur charbon actif en poudre en présence de tensioactifs, ions calcium et bichromate} Essai de modélisation

\section{Sorption of atrazine and diuron on powdered activated carbon with surfactants, calcium and dichromate ions}

Try of modelling

\section{A. YAACOUBI ${ }^{1}$ et J. AYELE2*}

Reçu le 25 juillet 1997, accepté le 20 novembre 1998*

\section{SUMMARY}

Contamination of natural waters by organic micropollutants like pesticides requires the use of powdered activated carbon (PAC) during the flocculation step of water treatment. One of the main factors affecting the efficiency of this process is the presence in natural waters of organic and mineral compounds. The aim of this study was to determine the influence of two surfactants [(i) anionic (sodium dodecyl sulfate: SDS) and (ii) cationic (hexadecyl-trimethyl ammonium bromide: HTAB)], and two mineral ions (calcium and dichromate) upon atrazine and diuron adsorption on powderd activated carbon resulting from Chemviron Filtrasorb-400. The adsorption capacities on PAC of atrazine and diuron alone were determinated according to the Langmuir model for the isothermes (table 1). They were estimated to be 1.42 and $1.72 \mathrm{mmol} \cdot \mathrm{g}^{-1}$ respectly at $\mathrm{pH}$ 5.5. Coadsorption results pointed out that the herbicide adsorption was inhibited in the presence of surfactants whatever their nature (figure $I$ and 2). This inhibition was most importante with HTAB $(45 \%)$, according to the zeta surface potential and the streric dimensions of molecules, than with SDS $(23 \%)$ for atrazine. The same result was noted for diuron with an inhibition respectly of $15 \%$ for SDS and $27 \%$ for HTBA. Calcium and dichromate ions, each one with one herbicide had no effect. In any case, Langmuir isothermes were drawn and adsortion capacities were calculated with a good correlation (tables 2 and 3 ). The influence of coadsorbats was always greatest for atrazine than for diuron. All the experiments were performed at pH $=5.5$, without influence upon the adsorption, even for the dichromate ions.

Desorption tests showed that a part of adsorbed pesticides was desorbed with surfactants introduction (figure 3): with SDS $12.3 \%$ of atrazine and $8 \%$ of diu-

1. Laboratoire de Chimie Organique Appliquée, Unité : Analyse et Environnement, Faculté des SciencesSemlalia, BPS 15, Marrakech, Maroc.

2. Laboratoire des Sciences de l'Eau et de l'Environnement, Faculté des Sciences, 123, avenue Albert Thomas 87060 Limoges, France.

* Correspondance.

* * Les commentaires seront reçus jusqu’au 30 décembre 1999. 
ron against respectly $23 \%$ and $17 \%$ with HTBA. The phenomenon was increased with SDS in presence of calcium ions (figures 4 and 5) according to the increase of SDS adsorption (table 2 and 3).

The use of two simple coadsorption models was reinforced by the calculated values obtained for adsoartion capacities (figures 6 and 7) which were very closely with the experimental ones.

The calculation of constantes given by the models confirmed the existence of specific sites of adsorption for every compound and a non competitive inhibition due to a change in the nature of the interactions between pesticides and activated carbon surface (table 3 ).

Key-words: adsorption, competition, powdered activated carbon, model, atrazine, diuron, surfactants, calcium and dichromate ions.

RÉSUMÉ

La contamination des eaux naturelles par les micropolluants organiques tels que les herbicides, nécessite le recours à des procédés très performants, en particulier l'adsorption sur charbon actif pour satisfaire aux normes de potabilité sur les eaux distribuées. Si le charbon actif en poudre (CAP) présente une bonne efficacité pour l'élimination des herbicides seuls dans l'eau distillée, la présence dans l'eau d'autres composés organiques et/ou minéraux aura pour conséquence la modification des paramètres de l'équilibre de l'adsorption du système initial et la modification des performances du matériau adsorbant. Cette étude a montré que le CAP utilisé présente une bonne affinité pour les deux herbicides étudiés (atrazine et diuron), dont les capacités d'adsorption, déterminées par le modèle de Langmuir, sont 1,42 et $1,72 \mathrm{mmol} \cdot \mathrm{g}^{-1}$ respectivement. La présence de composés organiques tels que les tensioactifs anionique le dodécylsulfate de sodium (DSS), et cationique le bromure d'hexadécyl triméthyl ammonium (BHTA) provoque une forte diminution de la capacité d'adsorption des deux herbicides par le charbon actif. Le BHTA provoque l'effet le plus marqué, par contre, les ions calcium et bichromate n'ont pas d'influence sur l'adsorption des deux herbicides. Les essais de désorption ont montré que l'introduction des coadsorbats organiques provoque la désorption d'une partie de l'herbicide. Ce phénomène est accentué dans le cas du DSS en présence d'ions calcium. L'application de modèles simples de coadsorption, a permis de mettre en évidence l'existence presque exclusive de sites spécifiques d'adsorption pour chacun des substrats étudiés et une inhibition non compétitive due a une interdépendance possible entre les sites d'adsorption et une altération des interactions de chaque composé à la surface du charbon

Mots clés : adsorption, compétition, charbon actif en poudre, modèle, atrazine, diuron, tensioactifs, ions calcium et bichromate.

\section{INTRODUCTION}

Les composés organiques contenus dans les eaux naturelles sont d'une part, responsables d'un certain nombre de problèmes, coloration et odeur notamment, et d'autre part, ils sont quelquefois toxiques pour l'être humain et l'environnement.

Parmi les principaux polluants liés à l'activité humaine, les pesticides tiennent une place importante. Les normes françaises actuelles pour l'eau potable, indiquent une quantité de $0,1 \mu \mathrm{g} \cdot \mathrm{L}^{-1}$ par substance isolées et $0,5 \mu \mathrm{g} \cdot \mathrm{L}^{-1}$ pour 
l'ensemble des pesticides. Dans une filière de production d'eau de consommation, si les étapes de floculation - décantation et filtration sur sable sont peu efficaces pour l'élimination des pesticides, d'autres traitements comme l'oxydation et l'adsorption sur charbon actif (PAILLARD et al., 1990; PRADOs et CIBA, 1997) ou certains procédés membranaires (MANDRA et al., 1993 ; BAUDIN et al., 1997) donnent de meilleurs résultats. Aujourd'hui, l'utilisation du charbon actif reste l'une des meilleures solutions pour l'élimination de la plupart des polluants organiques (GAID et al., 1994 ; AYELE et al., 1996 ; WELTE et al., 1996).

L'atrazine, herbicide de la famille des S-triazines, a été utilisée intensivement jusqu'à ces dernières années surtout en agriculture, et reste actuellement l'un des principaux pesticides retrouvés dans les eaux (MAUPAS et GILLET, 1992 ; SELIM et WANG, 1994 ; PRADOS et CIBA, 1997). Elle apparaît comme un bon indicateur de la pollution puisqu'on ne trouve pas d'autres pesticides là où sa concentration reste modérée. Actuellement, on s'intéresse de plus en plus aux métabolites de l'atrazine qui se retrouvent en quantité non négligeable dans toutes les eaux à potabiliser qui contiennent la molécule mère (PRADOS et CIBA, 1997). Le diuron, quant à lui, a vu son utilisation progresser pour des usages principalement non agricoles (entretien des voies de transport et des espaces urbains, etc.). Il se retrouve actuellement dans de nombreuses eaux naturelles.

La formulation commerciale d'un pesticide intègre l'adjonction d'adjuvants notamment des tensioactifs. Ces produits transportés par les eaux constituent une source de pollution pour les sols, les nappes et les eaux de surface. Mais les tensioactifs provenant de l'utilisation de détergents et d'agents de surface représentent la principale source de pollution par ces produits.

Si le charbon actif en poudre (CAP) présente une bonne capacité d'adsorption pour les pesticides seuls dans l'eau distillée, la présence dans les eaux naturelles d'autres composés organiques et minéraux aura pour conséquence la modification des paramètres de l'équilibre de l'adsorption du système initial et la modification des performances du matériau adsorbant.

Lors du traitement des eaux naturelles pour la production d'eau potable, différents composés organiques se retrouvent donc en compétition pour l'adsorption sur charbon actif. Cette compétition provoque une diminution de l'efficacité du charbon actif face à une pollution donnée (YAACOUBl et al., 1991 ; SOUABI et al., 1994 ; AYELE et al., 1995 ; GICQUEL et al., 1997).

La présence de sels métalliques peut modifier l'adsorption de certains composés organiques, essentiellement ioniques, par un changement des propriétés physico-chimiques des solutés et/ou la modification des propriétés de la surface du charbon actif (RANDTKE et JEPSEN, 1982 ; LAFRANCE et MAZET, 1985 ; MAZET et al., 1988).

Afin de mieux appréhender le comportement de ces composés sur charbon actif, une étude au laboratoire s'avère nécessaire. Dans ce travail, nous nous proposons d'étudier l'adsorption de deux herbicides (''atrazine et le diuron) sur CAP en présence de coadsorbats organiques (tensioactifs anionique et cationique) et minéraux (ions calcium et ions bichromate). Les résultats obtenus permettront de discuter et d'approcher les mécanismes de l'adsorption de ces solutés seuls ou en mélange sur le charbon actif.

L'utilisation de modèles mathématiques simples permettra de confirmer les hypothèses émises lors de l'adsorption. 


\section{MATÉRIELS ET MÉTHODES}

\section{Matériau adsorbant}

Le charbon actif en poudre utilisé est le Chemviron Filtrasorb-400, d'origine minérale, activé à haute température en présence d'oxygène. C'est un charbon mésoporeux, sa surface spécifique déterminée par l'adsorption de l'azote et l'isotherme (BET) est de $1050 \mathrm{~m}^{2} \cdot \mathrm{g}^{-1}$. II reste un des charbons les plus employés dans les usines de production d'eau potable, et il présente de bonnes performances par rapport aux autres charbons actifs.

Le CAP obtenu après broyage et tamisage, de granulométrie égale ou inférieure à $50 \mu \mathrm{m}$, est traité avec une solution d'acide chlorhydrique $1 \mathrm{~N}$ pendant $72 \mathrm{~h}$ en flacon fermé et agité ; il est ensuite filtré et rincé avec de l'eau distillée jusqu'à pH constant $(5,5)$, puis séché pendant $24 \mathrm{~h}$ à $105^{\circ} \mathrm{C}$ et conservé en flacon fermé. Ce traitement permet d'éliminer les ions fixés à la surface et de mieux appréhender le seul phénomène d'adsorption.

\section{Produits utilisés}

Les herbicides sont :

- L'atrazine : 2-chloro 4-N-éthylamino 6-N-isopropylamino S-triazine (Chem service, France), de pureté $99,5 \%$, son seuil de solubilité dans l'eau est de $35 \mathrm{mg} \cdot \mathrm{L}^{-1}$ à $20^{\circ} \mathrm{C}$.

- Le diuron : 3-(3,4-dichlorophényl)-1,1-diméthylurée (Chem service, France), de pureté $98 \%$, son seuil de solubilité dans l'eau est de $42 \mathrm{mg} \cdot \mathrm{L}^{-1}$ à $20{ }^{\circ} \mathrm{C}$.

Les tensioactifs sont:

- Le dodécylsulfate de sodium (DSS): $\mathrm{CH}_{3}\left(\mathrm{CH}_{2}\right)_{11} \mathrm{SO}_{4} \mathrm{Na}$ (Aldrich), de pureté $98 \%$.

- Le bromure d'hexadécyltriméthylammonium (BHTA): $\mathrm{CH}_{3}\left(\mathrm{CH}_{2}\right)_{15} \mathrm{~N}\left(\mathrm{CH}_{3}\right)_{3} \mathrm{Br}$ (Fluka company), de pureté supérieure à $98 \%$. $99 \%$.

- Le chlorure de calcium : $\mathrm{CaCl}_{2}, 2 \mathrm{H}_{2} \mathrm{O}$ (R.P. Prolabo) de pureté supérieure à

- Le bichromate de potassium : $\mathrm{K}_{2} \mathrm{Cr}_{2} \mathrm{O}_{7}$ (R.P. Prolabo) de pureté supérieure à $99,5 \%$.

Des solutions mères ont été préparées dans l'eau distillée, et les solutions filles de concentrations désirées sont obtenues par dilution.

\section{Méthodes d'analyses}

L'atrazine et le diuron ont été dosés par chromatographie liquide haute pression (HPLC), à l'aide d'un appareillage constitué d'une pompe L6000 Hitachi, d'une colonne RP18 (silice greffée, Licrospher, $5 \mu \mathrm{m}, 25 \mathrm{~cm}$ ), d'un détecteur UV L400, d'un intégrateur Hitachi D2500 et d'un passeur automatique d'échantillons Hitachi. La phase éluante est un mélange méthanol/eau (80/20), elle a été préalablement dégazée sous vide. Le débit d'élution est de $0,5 \mathrm{~mL} \cdot \mathrm{min}^{-1}$, le volume d'injection est de $50 \mu \mathrm{L}$ pour l'atrazine et $100 \mu \mathrm{L}$ pour le diuron, les longueurs d'onde utilisées pour le détecteur UV sont 222 et $248 \mathrm{~nm}$ respectivement.

Aucune interférence, dans le dosage par HPLC des deux herbicides, n'a été observée en présence d'autres coadsorbats. 


\section{Mode opératoire}

Toutes les solutions sont préparées à partir d'eau distillée $(\mathrm{pH}=5,5$; $\chi=2 \mu \mathrm{S} \cdot \mathrm{cm}^{-1}$ ).

Essais d'adsorption: Ils sont menés en réacteur discontinu, à une température de $20^{\circ} \mathrm{C}( \pm 2)$ sur des volumes de $100 \mathrm{~mL}$ continuellement agités (secoueur, 1000 coups $/ \mathrm{min}$ ) en présence de quantités variables de CAP $(\mathrm{m})$ et d'adsorbats à la concentration initiale $\left(C_{0}\right)$. Aucune variation de $\mathrm{pH}$ n'a été observée lors de la mise en suspension des faibles quantités de CAP utilisé, ainsi qu'au terme des essais d'adsorption.

Lorsque le temps de contact (déterminé par des essais cinétiques) est écoulé, les suspensions de CAP sont filtrées sur nitrate de cellulose $0,3 \mu \mathrm{m}$ et le filtrat est dosé pour évaluer la concentration résiduelle $\left(C_{r}\right)$ de pesticide en solution, la concentration adsorbée $\left(C_{a d}\right)$ est obtenue par différence : $C_{a d}=C_{0}-C_{r}$

Des essais témoins ont été effectués pour chaque mélange et n'ont montré aucune perte détectable des adsorbats par complexation et/ou filtration pour les concentrations utilisées.

Tous les résultats représentés sont obtenus à partir de la moyenne d'au moins 3 essais.

Essais de désorption: Les essais de désorption des pesticides en présence de différents coadsorbats ont été réalisés comme suit :

- Une masse de $500 \mathrm{mg}$ de CAP, dans un litre d'eau distillée contenant le pesticide (à la limite de solubilité) est agitée pendant $5 \mathrm{~h}$. Après filtration, on sèche le CAP à $90^{\circ} \mathrm{C}$ pendant $24 \mathrm{~h}$; ce charbon sera utilisé après refroidissement pour réaliser les essais de désorption.

- Une masse de $10 \mathrm{mg}$ de CAP précédemment " saturé " avec le pesticide est mise en contact avec une solution contenant le coadsorbat ( $100 \mathrm{~mL}$ ). Après $3 \mathrm{~h}$ d'agitation, on filtre et on détermine la concentration de pesticide désorbé. Des essais témoins ont permis de déterminer la quantité de pesticide désorbée dans l'eau distillée.

\section{Modèles mathématiques}

Modèle de Langmuir pour. un soluté seul : Les résultats de l'adsorption sont exploités sous forme d'isothermes à l'aide du modèle de Langmuir sous ses deux formes, fonction de saturation et forme linéarisée (Stumm et Morgan, 1981)

$$
\Gamma=\Gamma^{\infty} \cdot K \cdot C_{r} /\left(1+K \cdot C_{r}\right) ; \quad 1 / \Gamma=1 / \Gamma^{\infty}+1 / \Gamma^{\infty} \cdot K \cdot C_{r}
$$

$\Gamma^{\infty}:(\mathrm{Cad} / \mathrm{m}) \max =$ Capacité maximale théorique d'adsorption en $\mathrm{mmol} / \mathrm{g}$

$\Gamma:(\mathrm{Cad} / \mathrm{m})=$ Capacité d'adsorption en $\mathrm{mmol} / \mathrm{g}$

$\mathrm{K}$ : Constante thermodynamique de l'équilibre d'adsorption

$\mathrm{C}_{\mathrm{r}}$ : Concentration résiduelle en soluté en $\mathrm{mol} \cdot \mathrm{L}^{-1}$

Modèle de coadsorption compétitive (DUSART, 1986). On suppose qu'il n'existe qu'un seul type de sites susceptibles de fixer les deux adsorbats (avec une cinétique propre à chacun) et que ces sites peuvent adsorber indifféremment l'un ou l'autre soluté. En fait la fixation de l'un inhibe la fixation de l'autre sur le même site. 
Un modèle simple permet le calcul de l'adsorption ultime qui aurait lieu de façon concurrentielle sur des sites identiques recouverts avec la même probabilité par les deux types de molécule La courbe idéale se traduit par:

$$
\Gamma_{\text {cal }}^{\infty}=X_{A} \cdot \Gamma_{\left(A^{\circ}\right)}^{\infty}
$$

Pour un composé $A, X_{A}$ est la fraction molaire de $A$ dans le mélange, $\Gamma_{\left(A^{\circ}\right)}^{\infty}$ la capacité d'adsorption maximale du composé $A$ seul et $\Gamma_{c a l}^{\infty}$ celle qu'il devrait avoir dans le mélange en présence d'un deuxième soluté $B$

Modèle de coadsorption mixte (DUSART, 1986). Dans ce cas, on suppose que les deux solutés $A$ et $B$ pourront se fixer de façon concurrentielle sur un certain nombre de sites, ainsi que sur des sites spécifiques dont l'accès pourra être modifié par la présence de l'autre substrat.

Si le composé $A$ est seul, ses sites d'adsorption peuvent se décomposer en deux parties : une partie qui pourra convenir aussi à $B$ et une fraction de sites spécifiques. L'adsorption ultime totale de $A$ seul $\Gamma_{\left(A^{\circ}\right)}^{\infty}$ correspondante sera :

$$
\Gamma_{\left(A^{\circ}\right)}^{\infty}=\gamma_{(A B)}+\gamma_{(A)}
$$

$\gamma_{(A B)}$ étant l'adsorption correspondant aux sites communs des solutés $A$ et $B$, $\gamma_{(A)}$, celle correspondant aux sites propres du soluté $A$.

Pour le mélange $(A+B)$, l'adsorption est supposée concurrentielle sur les sites communs aux deux solutés donc $\gamma_{(A B)}$ est réduit en $X_{A} \cdot \gamma_{(A B)}, X_{A}$ étant la fraction molaire de $A$ dans le mélange. Quant à l'adsorption sur les sites propres, elle est soit diminuée, soit augmentée par la création de sites supplémentaires. Ceci se traduit par l'affectation au terme $\gamma_{(A)}$, d'une fonction exponentielle, $\exp \left(a \cdot C_{B}\right)$ où a représente un paramètre qui, selon son signe, traduira l'influence de $B$ : soit une inhibition de l'adsorption de $A(a<0)$, soit une promotion $(a>0)$ ou une absence d'influence $(a=0) . C_{B}$ représente la concentration de $B$ dans le mélange.

L'adsorption ultime totale de A s'écrit alors :

$$
\Gamma_{(A)}^{\infty}=X_{A} \cdot \gamma_{(A B)}+\gamma_{(A)} \exp \left(a \cdot C_{B}\right)
$$

$\Gamma_{(A)}^{\infty}$ étant la capacité d'adsorption maximale de $A$ dans le mélange.

L'introduction du terme i permet de lier les sites propres et communs :

$$
i=\gamma_{(A)} \gamma^{\prime} \gamma_{(A B)} \quad \text { avec } i[0,+\infty]
$$

si $\quad \mathrm{i}=0$ : il n'y a pas de sites propres à $A$;

$i \geq 10^{3}$ : il n'y aura pratiquement pas de sites communs à $A$ et $B$.

\section{RÉSULTATS ET DISCUSSIONS}

\section{Résultats expérimentaux d'adsorption}

Les concentrations initiales $\left(C_{0}\right)$ des solutés sont fixées à $50 \mu \mathrm{mol} \cdot \mathrm{L}^{-1}$ pour l'atrazine et $100 \mu \mathrm{mol} \cdot \mathrm{L}^{-1}$ pour le diuron. Ces concentrations, plus de mille fois supérieures à celles rencontrées dans les eaux naturelles, permettent d'utiliser 
des doses de CAP comprises entre 20 et $80 \mathrm{mg} \cdot \mathrm{L}^{-1}$ et d'avoir des concentrations résiduelles en pesticides facilement dosables, pour le tracé des isothermes.

Les cinétiques d'adsorption de l'atrazine, du diuron, du DSS et du BHTA, seuls ou en présence de coadsorbats, ont montré que le temps nécessaire pour atteindre l'équilibre d'adsorption est de $3 \mathrm{~h}$, quel que soit le mélange utilisé. $\mathrm{Ce}$ temps a été adopté pour tous les essais d'isothermes.

\section{Adsorption des solutés seuls}

L'adsorption des tensioactifs sur charbon actif montre que le BHTA s'adsorbe mieux que le DSS. La mesure du potentiel zêta, en relation avec la charge de surface des particules de CAP seules dans l'eau distillée, est de $-20 \mathrm{mV}$; il augmente vers les valeurs positives avec la fixation du BHTA, et devient plus négatif avec l'adsorption du DSS (tableau 1).

Tableau 1 Caractéristiques de l'adsorption, selon le modèle de Langmuir, des solutés seuls sur CAP.

Table 1 Adsorption characteristics, according to the Langmuir model, of different solutes alone on PAC.

\begin{tabular}{|c|c|c|c|c|c|}
\hline & CAP seul & DSS & BHTA & Atrazine & Diuron \\
\hline $\begin{array}{l}\Gamma^{\infty} \\
\left( \pm 0,02 \mathrm{mmol} \cdot \mathrm{g}^{-1}\right)\end{array}$ & & 0,50 & 0,70 & 1,42 & 1,71 \\
\hline $\begin{array}{l}K \\
(\mu \mathrm{M})^{-1}\end{array}$ & & 1,05 & 2,30 & 1,09 & 1,07 \\
\hline $\begin{array}{l}\text { Potentiel. zêta } \\
( \pm 1 \mathrm{mv})\end{array}$ & -20 & -30 & -4 & -21 & -20 \\
\hline
\end{tabular}

Ceci indique que l'adsorption, dans ces deux cas, se fait majoritairement par un mécanisme hydrophobe mettant en jeu les segments apolaires des tensioactifs et les sites hydrophobes du CAP. L'hydrophobie de ces molécules augmente avec l'allongement de la chaîne linéaire qui augmente la surface disponible pour des interactions de type Van Der Waals, mais aussi à cause des répulsions entre la surface du CAP chargée négativement et le groupement sulfate du DSS.

Les deux pesticides, molécules peu solubles et contenant des cycles aromatiques, ne sont pas ionisés à $\mathrm{pH}=5,5$ et leur capacité d'adsorption est de $1,42 \mathrm{mmol} \cdot \mathrm{g}^{-1}$ pour l'atrazine et $1,72 \mathrm{mmol} \cdot \mathrm{g}^{-1}$ pour le diuron. La faible taille de ces pesticides diminue leur encombrement stérique et ces molécules peuvent avoir accès à des pores de faible volume. II semble aussi que le mécanisme mis en jeu soit de nature hydrophobe, par l'intermédiaire, des interactions de type Van Der Waals et des liaisons hydrogène avec les fonction de surface du CAP d'une part, et des interactions de type $\pi-\pi$ entre les noyaux aromatiques grâce à une affinité supplémentaire liée à la structure aromatique de l'adsorbant et de l'adsorbat d'autre part.

\section{Adsorption des herbicides en présence des tensioactifs et d'ions calcium et bichromate}

Les isothermes d'adsorption selon le modèle de Langmuir de l'atrazine et du diuron sont réalisées en présence des deux tensioactifs (DSS et BHTA); les résultats sont rassemblés sur les figures 1 et 2 et dans les tableaux 2 et 3 . Ils montrent que : 
a)

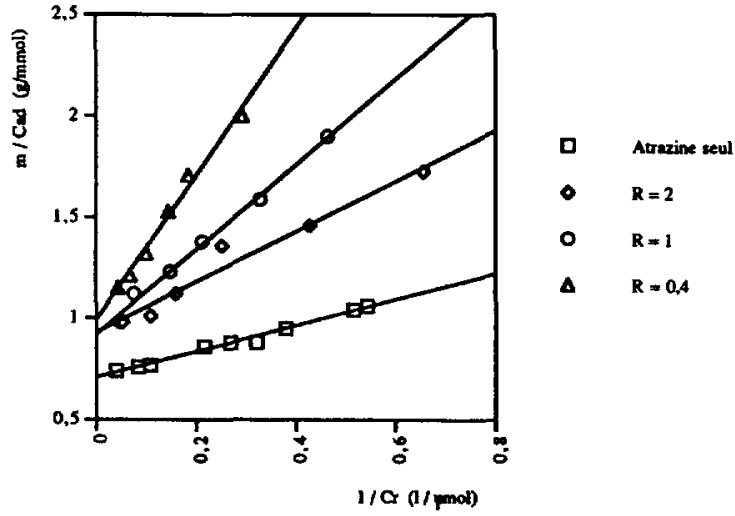

b)

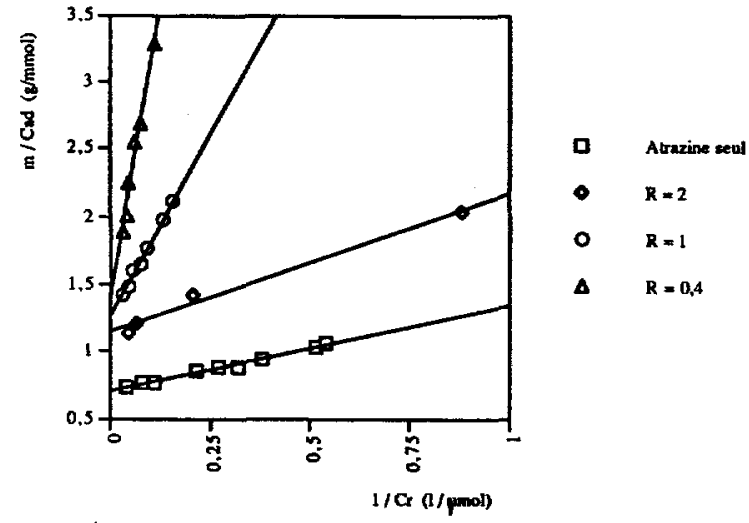

Figure 1 Isothermes d'adsorption (Langmuir) de l'atrazine en présence de concentration croissantes en tensioactif. $\mathrm{C}_{\mathrm{o}}=50 \mu \mathrm{mol} \cdot \mathrm{L}^{-1}, \mathrm{pH}=5,5, \mathrm{R}=$ atrazine/ tensioactif.
a) DSS.

b) BHTA.

Atrazine adsorption isotherms (Langmuir) with increasing surfactant concentrations $C_{0}=50 \mu \mathrm{mol} \cdot \mathrm{L}^{-1}, \mathrm{pH}=5,5, R=$ atrazine $/$ surfactant.
a) SDS.
b) HTAB.

- le BHTA diminue l'adsorption des deux herbicides de façon beaucoup plus importante que le DSS ;

- quelle que soit la nature du tensioactif en compétition d'adsorption avec ces herbicides, les capacités d'adsorption par le charbon actif de ces derniers diminuent lorsque la concentration du tensioactif augmente.

Des résultats précédents ont montré qu'il en était de même avec un tensioactif non ionique (AYELE et al., 1995) et avec la matrice organique naturelle exprimée en COT, $\mathrm{DO}_{254 \mathrm{~nm}}$ et oxydabilité au $\mathrm{KMnO}_{4}$ (GICQUEL et al., 1997). 
a)

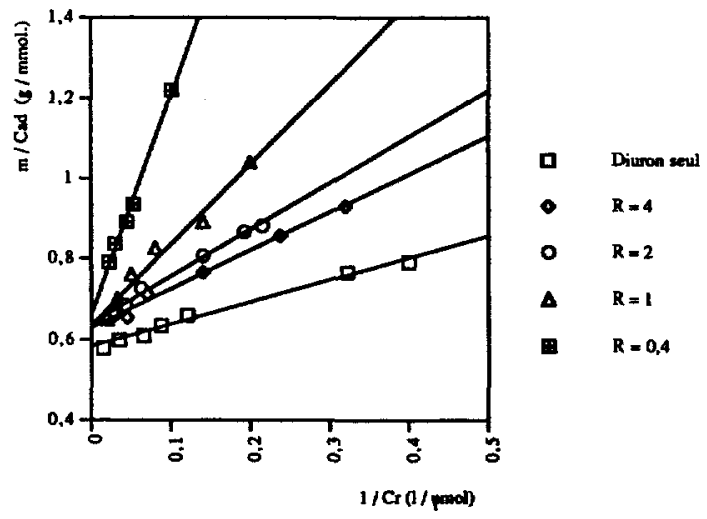

b)

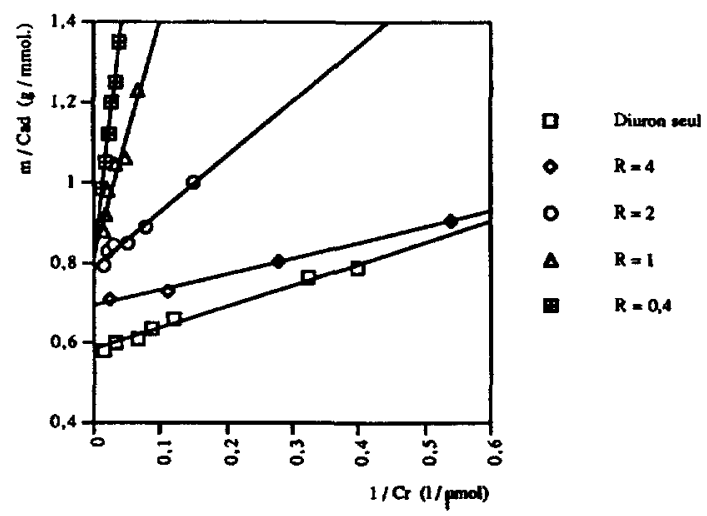

Figure 2 Isothermes d'adsorption (Langmuir) du diuron en présence de concentration croissantes en tensioactif. $\mathrm{C}_{0}=100 \mu \mathrm{mol} \cdot \mathrm{L}^{-1} ; \mathrm{pH}=5,5 ; \mathrm{A}=$ diuron/ tensioactif.

a) DSS.

b) BHTA.

Diuron adsorption isotherms (Langmuir) with increasing surfactant concentrations. $C_{0}=50 \mu \mathrm{mol} \cdot \mathrm{L}^{-1}, \mathrm{pH}=5,5, R=$ diuron/surfactant.

a) $S D S$.

b) $H T A B$.

Les valeurs de la capacité maximale d'adsorption $\left[(\mathrm{Cad} / \mathrm{m})_{\max }=\Gamma^{\infty}\right]$, obtenues à saturation, en présence de différentes concentrations en tensioactifs anionique et cationique sont rassemblées dans les tableaux 2 et 3.

On remarque que pour une concentration croissante en tensioactif la valeur de la capacité maximale d'adsorption diminue fortement jusqu'à l'obtention d'un rapport molaire $(R=$ pesticide/tensioactif $) R=1$; au-delà de ce rapport, l'excès de la concentration en tensioactif prolonge l'inhibition de façon très faible. Ainsi en l'absence de coadsorbat, on obtient une capacité d'adsorption de $1,42 \mathrm{mmol} \cdot \mathrm{g}^{-1}$ 
Tableau 2 Variation des paramètres d'adsorption de l'atrazine selon le modèle de Langmuir en présence de tensioactifs et d'ions calcium et bichromate.

$\mathrm{R}=$ [herbicide] $\left[\right.$ [tensioactif] ; $\mathrm{pH}=5,5 ; \mathrm{Co}=50 \mu \mathrm{mol} \cdot \mathrm{L}^{-1}$.

Table 2 Variation of adsorption parameters of atrazine, according to the Langmuir model, in presence of surfactants, calcium and dichromate ions.

$A=[$ pesticide $][$ surfactant $] ; \mathrm{pH}=5,5 ; \mathrm{Co}=50 \mu \mathrm{mol} \cdot \mathrm{L}^{-1}$.

\begin{tabular}{|c|c|c|c|c|c|c|c|c|}
\hline R & & & seul & 8 & 4 & 2 & 1 & 0,4 \\
\hline \multirow{3}{*}{$\begin{array}{l}\text { Atrazine } \\
+ \\
\text { DSS }\end{array}$} & $\Gamma^{\infty}$ & Saturation & 1,45 & 1,27 & 1,23 & 1,15 & 1,09 & 0,96 \\
\hline & $\left(\mathrm{mmol} \cdot \mathrm{g}^{-1}\right)$ & $\begin{array}{l}\text { Forme } \\
\text { linéarisée }\end{array}$ & 1,42 & 1 & 1 & 1,08 & 1,09 & 1,01 \\
\hline & $\mathrm{K}(\mu \mathrm{M})^{-1}$ & & 1,09 & 1 & 1 & 0,74 & 0,43 & 0,27 \\
\hline \multirow{3}{*}{$\begin{array}{l}\text { Atrazine } \\
+ \\
\text { BHTA }\end{array}$} & $\Gamma^{\infty}$ & Saturation & 1,45 & 1,25 & 1,09 & 0,90 & 0,78 & 0,65 \\
\hline & $\left(\mathrm{mmol}^{-\mathrm{g}^{-1}}\right)$ & $\begin{array}{l}\text { Forme } \\
\text { linéarisée }\end{array}$ & 1,42 & 1 & 1 & 0,87 & 0,78 & 0,69 \\
\hline & $\overline{K(\mu \mathrm{M})^{-1}}$ & & 1,09 & 1 & 1 & 1,11 & 0,24 & 0,08 \\
\hline $\begin{array}{l}\text { Atrazine } \\
+ \\
\text { ions calcium }\end{array}$ & $\Gamma^{\infty}\left(\mathrm{mmol} \cdot \mathrm{g}^{-1}\right)$ & Saturation & 1,45 & 1 & 1 & 1,39 & $1, \overline{34}$ & 1,34 \\
\hline $\begin{array}{l}\text { Atrazine } \\
+ \\
\text { Bichromate }\end{array}$ & $\begin{array}{l}\Gamma^{\infty} \\
\left(\mathrm{mmol} \cdot \mathrm{g}^{-1}\right)\end{array}$ & Saturation & 1,45 & 1,52 & 1,53 & 1,60 & 1,50 & 1,45 \\
\hline
\end{tabular}

Tableau 3 Variation des paramètres d'adsorption du diuron, selon le modèle de Langmuir, en présence de tensioactifs et d'ions calcium et bichromate.

$\mathrm{R}=$ [herbicide] [tensioactif] $; \mathrm{pH}=5,5 ; \mathrm{Co}=100 \mu \mathrm{mol} \cdot \mathrm{L}^{-1}$.

Table 3 Variation of adsorption parameters of diuron, according to the Langmuir model, in presence of surfactants, calcium and dichromate ions.

$R=\left[\right.$ pesticide $/[$ surfactant $] ; p H=5,5 ; C o=50 \mu \mathrm{mol} \cdot \mathrm{L}^{-1}$.

\begin{tabular}{|c|c|c|c|c|c|c|c|c|}
\hline $\mathbf{R}$ & & & seul & 8 & 4 & 2 & 1 & 0,4 \\
\hline \multirow{3}{*}{$\begin{array}{l}\text { Diuron } \\
+ \\
\text { DSS }\end{array}$} & $\Gamma^{\infty}$ & Saturation & 1,72 & 1,69 & 1,57 & 1,48 & 1,46 & 1,32 \\
\hline & $\left(\mathrm{mmol}-\mathrm{g}^{-1}\right)$ & $\begin{array}{l}\text { Forme } \\
\text { linéarisée }\end{array}$ & 1,71 & 1 & 1,59 & 1,57 & 1,58 & 1,51 \\
\hline & $\overline{K(\mu M)^{-1}}$ & & 1,07 & $T$ & 0,66 & 0,55 & 0,32 & 0,12 \\
\hline \multirow{3}{*}{$\begin{array}{l}\text { Diuron } \\
+ \\
\text { BHTA }\end{array}$} & $\Gamma+\infty$ & Saturation & 1,72 & 1,67 & 1,49 & 1,40 & 1,26 & 1,19 \\
\hline & $\left(\mathrm{mmol}^{\left.-\mathrm{g}^{-1}\right)}\right.$ & $\begin{array}{l}\text { Forme } \\
\text { linéarisée }\end{array}$ & 1,71 & 1 & 1,45 & 1,27 & 1,22 & 1,24 \\
\hline & $\overline{K(\mu M)^{-1}}$ & & 1,07 & 7 & 1,77 & 0,57 & 0,14 & 0,06 \\
\hline $\begin{array}{l}\text { Diuron }+ \\
\text { Calcium }\end{array}$ & $\begin{array}{l}\Gamma^{\infty} \\
\left(\mathrm{mmol} \cdot \mathrm{g}^{-1}\right)\end{array}$ & Saturation & 1,72 & 1 & 1,72 & 1,81 & 1,66 & 1,7 \\
\hline $\begin{array}{l}\text { Diuron + } \\
\text { Bichromate }\end{array}$ & $\begin{array}{l}\Gamma^{\infty} \\
\left(\mathrm{mmol} \cdot \mathrm{g}^{-1}\right)\end{array}$ & Saturation & 1,72 & 1 & 1,73 & 1,8 & 1,73 & 1,75 \\
\hline
\end{tabular}

pour l'atrazine et de $1,72 \mathrm{mmol} \cdot \mathrm{g}^{-1}$ pour le diuron, tandis qu'en présence d'une concentration équimolaire en DSS et en BHTA on obtient pour l'atrazine une inhibition de $23 \%$ en présence du DSS contre $45 \%$ en présence du BHTA. 
Dans le cas du diuron l'effet des deux tensioactifs est faible, $15 \%$ d'inhibition pour le DSS et $27 \%$ pour le BHTA : le tensioactif cationique provoque un effet presque double de celui du DSS.

Cette différence entre ces deux coadsorbats pourrait provenir :

- d'une part de l'affinité entre ces tensioactifs et la surface du charbon : le tensioactif cationique s'adsorbe mieux que l'anionique, provoquant ainsi une inhibition de l'adsorption des herbicides due à une interdépendance possible entre les sites d'adsorption et/ou une altération des interactions de chaque composé à la surface du charbon (encombrement stérique, déstabilisation des liens entre les molécules de pesticides et la surface du charbon);

- d'autre part, du fait que l'adsorption des molécules chargées et leur disposition à la surface du CAP, peuvent agir sur l'adsorption des molécules d'herbicides, en générant des forces électrostatiques qui modifient l'approche de ces dernières vers la surface du charbon actif.

Le BHTA provoque plus d'encombrement stérique et il gène davantage l'adsorption des herbicides, soit par l'adsorption sur des sites adjacents aux sites du pesticide, soit par un blocage des pores dû à la grande taille de sa molécule.

Les ions calcium et bichromate ont très peu d'influence sur l'adsorption des deux herbicides, puisqu'ils ne modifient pas leur capacité d'adsorption aux erreurs expérimentales près (tableaux 2 et 3 ). Les ions calcium sont en effet déterminant du potentiel zêta des particules de CAP qu'ils peuvent neutraliser en favorisant ainsi l'adsorption de composés chargés négativement (MAZET et al., 1988). Mais dans le cas de molécules non chargées, le potentiel négatif du CAP n'a pas d'influence déterminante sur leur fixation. Par conséquent, les ions calcium n'ont pas d'influence sur l'adsorption des deux pesticides.

Par contre, l'étude du comportement des ions bichromate, dans la gamme du $\mathrm{pH}$ utilisée, montre une faible adsorption sur le charbon actif $\left(\Gamma^{\infty}=0,37 \mathrm{mmol} \cdot \mathrm{g}^{-1}\right.$ à $\mathrm{pH}=5,5$ ), résultats en accord avec ceux de PEREZ-CANDELA et al. (1995) et SHARMA et FORSTER (1996). Ces derniers auteurs ont montré que ladsorption est très influencée par le $\mathrm{pH}$ : l'élimination du chrome est favorisée pour des valeurs de $\mathrm{pH}$ très faibles $\left(\Gamma^{\infty}=0,57 \mathrm{mmol} \cdot \mathrm{g}^{-1}\right.$ à $\left.\mathrm{pH}=3,0\right)$. II semble que le mécanisme mis en jeu fasse intervenir les fonctions de surface du charbon, par conséquent pour le $\mathrm{pH}$ utilisé dans ce travail, la faible adsorption des ions bichromate ne gène pas l'adsorption des deux herbicides.

Le suivi de l'évolution des constantes thermodynamiques de l'adsorption (tableaux 2 et 3) montre qu'elles diminuent avec l'augmentation de la concentration en tensioactif, cette diminution est plus marquée en présence du BHTA, ce qui devrait favoriser la réaction de désorption des herbicides en présence de ces coadsorbats. Afin de vérifier cette hypothèse, des essais de désorption sont donc réalisés.

\section{Essais de désorption}

Les résultats (figure 3) montrent qu'une partie des pesticides est désorbée du CAP lorsqu'on introduit les tensioactifs et que le BHTA provoque l'effet le plus marqué.

Dans le cas de l'atrazine on obtient $12,5 \%$ de désorption en présence du DSS contre $23 \%$ en présence du BHTA, pour le diuron on a respectivement $8 \%$ 
a)

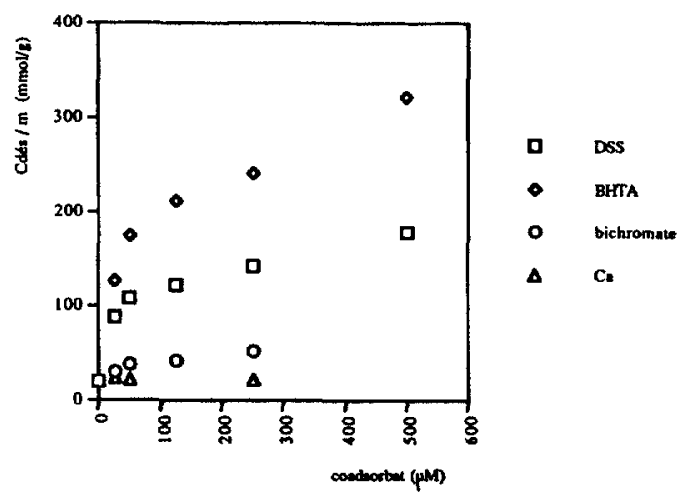

b)

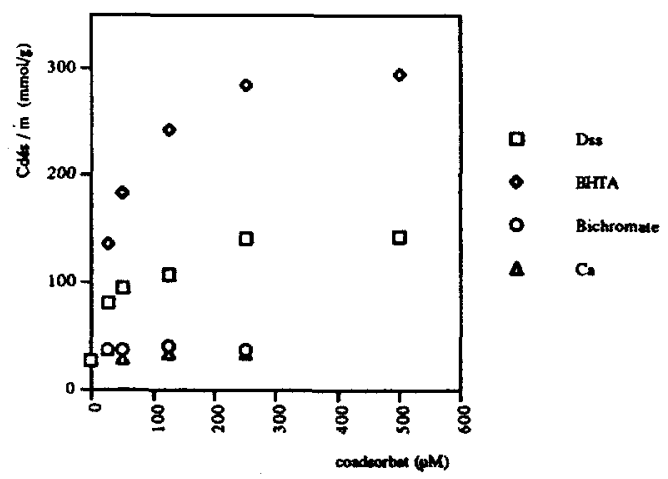

Figure 3 Désorption de pesticides en présence de tensioactifs.

a) Atrazine.

b) Diuron.

Pesticides desorption in presence of surfactants.

a) Atrazine.

b) Diuron.

et $17 \%$ de désorption. Ces résultats évoluent dans le même sens que ceux obtenus lors des essais d'adsorption, le tensioactif cationique provoque un effet double de celui de l'anionique. Les ions calcium et bichromate n'ont pas d'effet.

Ces résultats sont confirmés par l'adsorption des herbicides en présence des tensioactifs et de composés minéraux, le mélange (BHTA et bichromate) n'a pas été étudié du fait de la formation de complexe entre eux mêmes à faibles concentrations.

Sur les figures 4 et 5 on a représenté l'effet des tensioactifs sur la désorption des pesticides en présence d'ions calcium. Ces figures montrent que, les ions calcium n'ont un effet qu'en présence du DSS. II a été montré précédemment que la présence de ces ions provoque une augmentation de l'adsorption du DSS par la formation de complexes interadsorbats (MAZET et al., 1988) ce qui permet l'adsorption d'une quantité supplémentaire du DSS et en conséquence une inhibition plus marquée de l'adsorption des herbicides: On peut noter par exemple les valeurs des capacités maximales d'adsorption $\Gamma^{\infty}=1,42 \mathrm{mmol} \cdot \mathrm{g}^{-1}$ pour l'atrazine seule contre 1,09 $\mathrm{mmol} \cdot \mathrm{g}^{-1}$ en présence du DSS dans un rapport molaire $\mathrm{R}=1$ soit $23 \%$ d'inhibition et 
a)

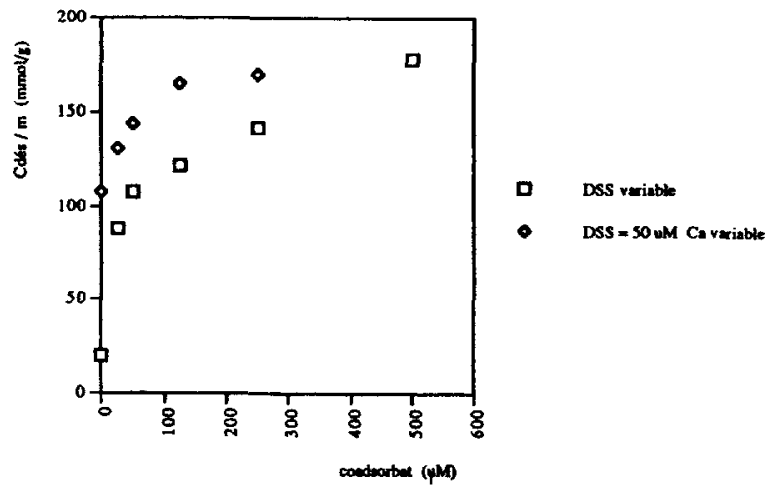

b)

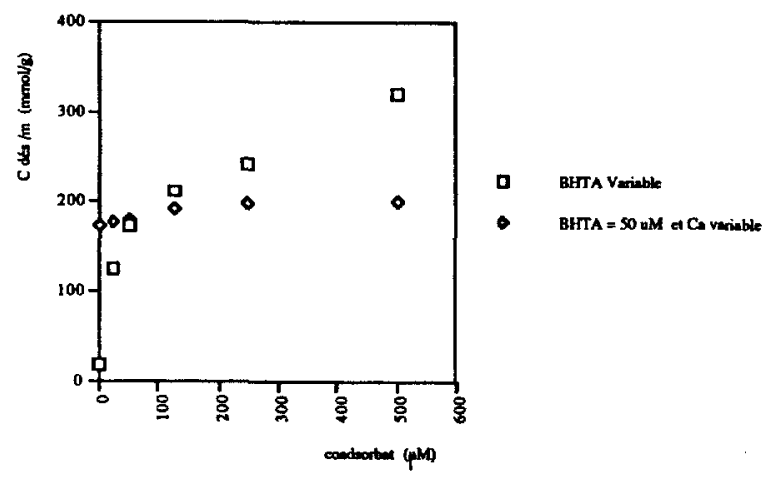

Figure 4 Effet de tensioactifs, en présence des ions calcium, sur la désorption de Patrazine.
a) DSS.
b) BHTA.

Effect of surfactants, in presence of calcium ions, on atrazine desorption.
a) $S D S$.
b) $H T A B$.

$0,80 \mathrm{mmol} \cdot \mathrm{g}^{-1}$ seulement en présence de DSS et de $\mathrm{Ca}^{2+}$ en concentrations équimolaires soit $44 \%$ d'inhibition. Pour le diuron on a respectivement, dans les mêmes conditions, $\Gamma^{\infty}=1,72 \mathrm{mmol} \cdot \mathrm{g}^{-1}, 1,36 \mathrm{mmol} \cdot \mathrm{g}^{-1}\left(15 \%\right.$ d'inhibition) et $1,32 \mathrm{mmol} \cdot \mathrm{g}^{-1}$ ( $23 \%$ d'inhibition). Ces résultats sont en accord avec les essais de désorption.

Le BHTA et les ions calcium n'ont pas d'effet sur la désorption des deux pesticides (figures $4 b$ et $5 b$ ).

\section{Essai de modélisation}

L'étude de l'évolution des paramètres de l'équilibre de l'adsorption de l'atrazine et du diuron en fonction de concentrations croissantes en tensioactifs peut nous permettre de discuter des mécanismes probables dans ce cas.

Sur les figures 6 et 7 , sont représentées les valeurs des capacités d'adsorption expérimentales et calculées à partir des modèles (I) et (III). Les valeurs calculées des paramètres i et a calculés sont présentées sur le tableau 4. 
a)

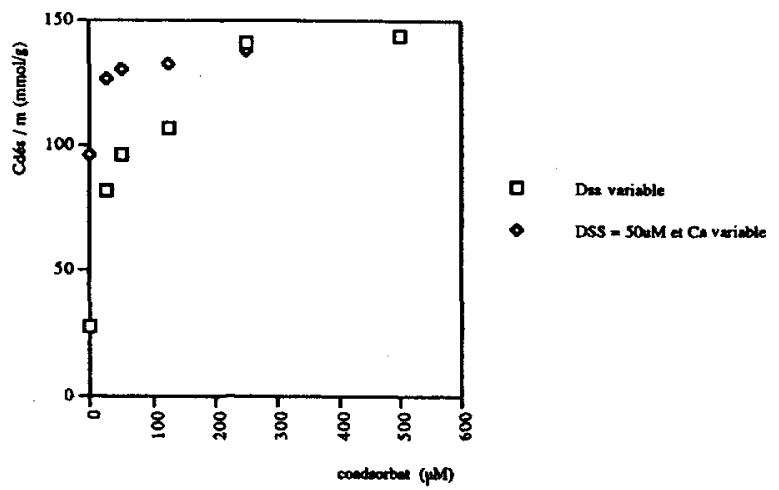

b)

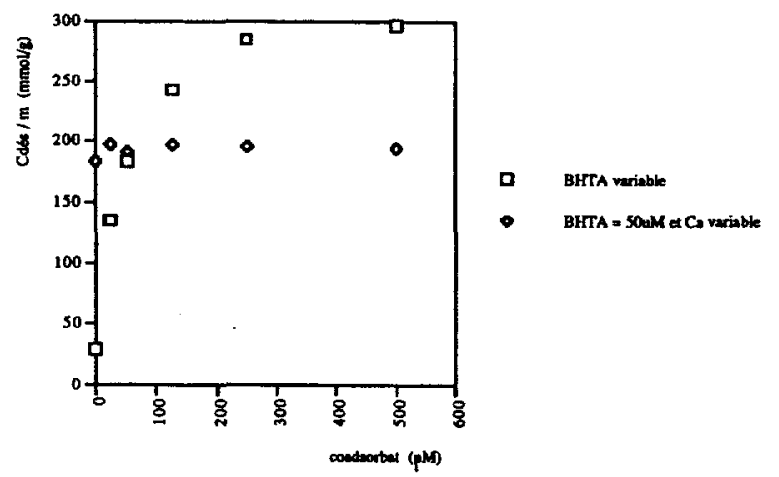

Figure 5 Effet des tensioactifs, en présence d'ions calcium, sur la désorption du diuron.

a) DSS.

b) BHTA.

Effect of surfactants, in presence of calcium ions, on diuron desorption.

a) SDS.

b) HTAB.

Les résultats, traités à l'aide du modèle (III) sont en bon accord avec l'expérience à l'erreur expérimentale près. Pour les quatre systèmes étudiés, le paramètre $\mathrm{i}$ est inférieur à $10^{3}$, ce qui signifie que la presque totalité des sites d'adsorption est spécifique. La valeur de a est négative, ce qui montre que les tensioactifs inhibent l'adsorption des herbicides probablement par une gène pour l'accès aux sites.

Les valeurs des constantes calculées confirment que le DSS a un effet moins important que celui du BHTA pour les deux pesticides; elles montrent aussi que les deux tensioactifs ont un effet plus marqué sur l'atrazine que sur le diuron. Ceci est en accord avec les résultats expérimentaux obtenus.

Linhibition de l'adsorption des deux herbicides serait due a une interdépendance possible entre les sites d'adsorption et une altération des interactions de chaque composé à la surface du charbon (encombrement stérique, déstabilisation des liens entre les molécules de pesticides et la surface du charbon). 
a)

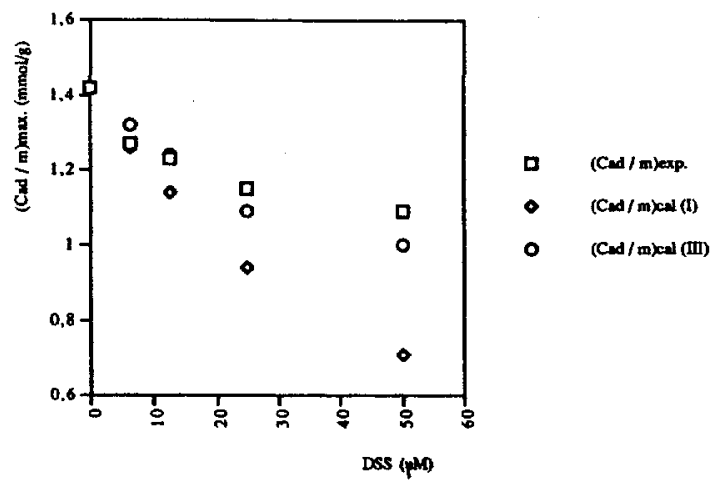

b)

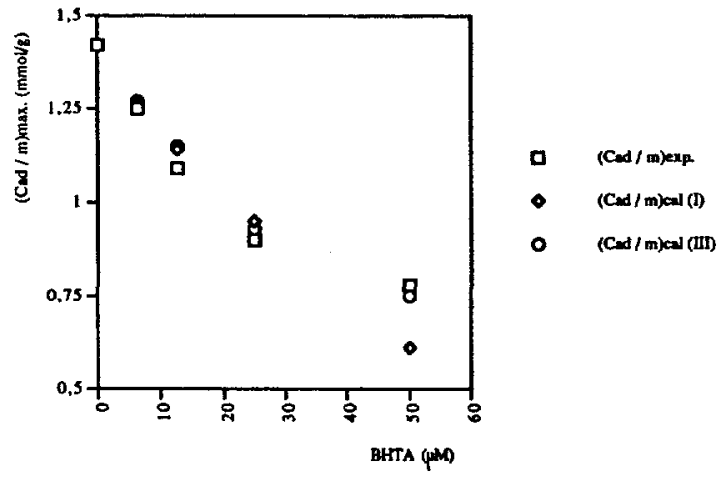

Figure 6 Variation de la capacité maximale d'adsorption (expérimentale et calculées) de l'atrazine en présence de concentrations croissantes en tensioactif.
a) DSS.
b) BHTA.

Change in atrazine adsorption capacity (experimental and calculed) with increasing surfactant concentrations.
a) $S D S$.
b) $H T A B$.

\section{CONCLUSION}

L'étude de l'influence de tensioactifs (DSS et BHTA) et des jons calcium et bichromate sur l'adsorption/désorption de l'atrazine et du diuron, sur charbon actif en poudre, en réacteur discontinu, a montré que :

Le charbon actif utilisé a une grande affinité pour les deux herbicides étudiés en raison de la faible taille de leurs molécules et des interactions favorables entre ces solutés et le matériau.

Quelle que soit la nature du tensioactif en compétition avec ces herbicides, la capacité d'adsorption de ces derniers, obtenue à partir du modèle de Langmuir, diminue lorsque la concentration en tensioactif augmente. 
a)

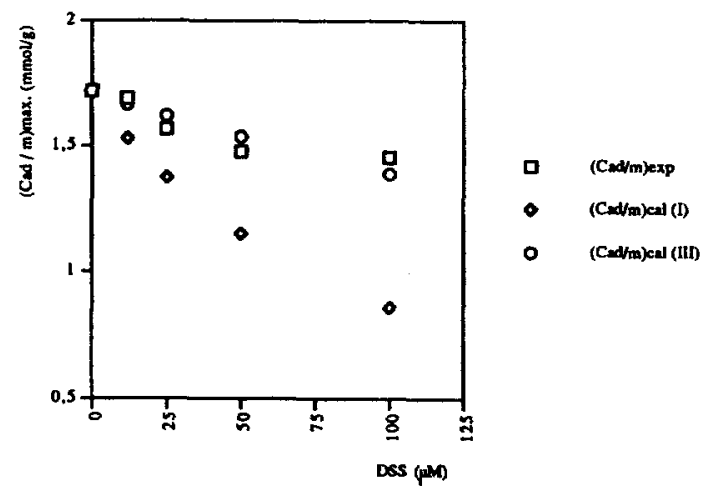

b)

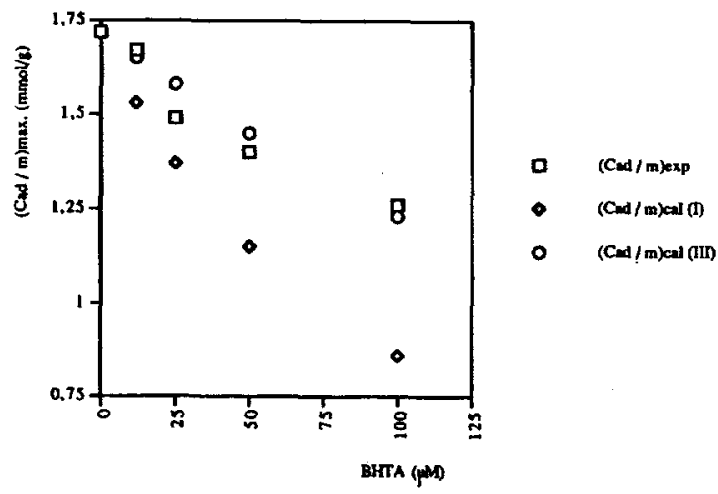

Figure 7 Variation de la capacité maximale d'adsorption (expérimentale et calculées) du diuron en fonction de la concentration en tensioactif.
a) DSS.
b) BHTA.

Change in the diuron adsorption capacity (experimental and calculed) with increasing surfactant concentrations.
a) SDS.
b) $H T A B$

Tableau 4 Caractéristiques de l'adsorption de l'atrazine et du diuron, selon les modèles l et III, en présence de concentrations variables en DSS et en BHTA.

Table 4 Adsorption characteristics of atrazine and diuron, according to models I and III, in presence of varying concentrations of SDS and HTAB.

\begin{tabular}{|lccccc|}
\hline \multicolumn{1}{|c}{ Mélange } & $\begin{array}{c}\Gamma^{\infty}\left({ }^{(\mathrm{A})}\right) \\
\left(\mathbf{m m o} \cdot \mathbf{g}^{-1}\right)\end{array}$ & $\mathbf{i}$ & $\begin{array}{c}\mathbf{a} \\
\left(\mathbf{I} \cdot \mathrm{mol}^{-1}\right)\end{array}$ & Sites & $\begin{array}{l}\text { Caractéristique } \\
\text { de l'adsorption }\end{array}$ \\
\hline $\begin{array}{l}\text { Atrazine } \\
+ \text { DSS variable }\end{array}$ & 1,42 & $\geq 10^{3}$ & $-1,0510^{4}$ & spécifiques & $\begin{array}{l}\text { inhibition } \\
\text { non compétitive }\end{array}$ \\
$\begin{array}{l}\text { Atrazine } \\
+ \text { BHTA variable } \\
\text { Diuron } \\
+ \text { DSS variable }\end{array}$ & 1,42 & $\geq 10^{3}$ & $-1,7110^{4}$ & spécifiques & $\begin{array}{l}\text { inhibition } \\
\text { non compétitive }\end{array}$ \\
$\begin{array}{l}\text { Diuron } \\
+ \text { BHTA variable }\end{array}$ & 1,72 & $\geq 10^{4}$ & $-2,1510^{3}$ & spécifiques & $\begin{array}{l}\text { inhibition } \\
\text { non compétitive }\end{array}$ \\
\hline
\end{tabular}


Le BHTA provoque plus d'inhibition que le DSS sur l'adsorption des deux herbicides étudiés. L'effet des deux tensioactifs est plus marqué sur l'atrazine. Les ions calcium et bichromate n'ont pas d'influence sur l'adsorption des deux herbicides. Ces résultats sont confirmés par les essais de désorption.

Dans le cas de l'adsorption (désorption) de l'atrazine et du diuron en présence de coadsorbats organiques et d'ions calcium, ces demiers ont un effet seulement en présence du DSS. Ils peuvent, en effet, modifier l'adsorption des composés organiques, essentiellement anioniques, et augmenter l'inhibition de l'adsorption de l'atrazine et du diuron.

L'essai de modélisation de la capacité maximale d'adsorption, obtenue à partir des isothermes de Langmuir, pour les mélanges herbicides - tensioactifs, en utilisant des modèles très simples, a permis de mettre en évidence l'existence presque exclusive de sites spécifiques d'adsorption pour chacun des substrat étudiés ainsi qu'une inhibition non compétitive due a une interdépendance possible entre les sites d'adsorption et une altération des interactions de chaque composé à la surface du charbon.

Par conséquent, lors du choix du charbon actif pour l'élimination d'un micropolluant donné, on doit tenir compte de ces phénomènes de compétition, fonction de la composition de l'eau traitée. La présence de coadsorbats a comme conséquence la diminution de l'efficacité du charbon actif.

NB : travail réalisé avec l'aide financière du Conseil Régional du Limousin.

\section{RÉFÉRENCES BIBLIOGRAPHIQUES}

AYELE J., LEVASSEUR P., MAZET M., 1996. Adsorption de triazines sur charbon actif en poudre, J. Water SRT-Aqua, 45, 28-34.

AYELE J., MAHI A., MAZET M., 1995. Étude de l'adsorption de l'atrazine sur charbon actif en poudre en présence de tensioactifs, Rev. Sci. Eau, 8, 355-370.

BAUDIN I., LAINE J.M., CHARLES P., DUGUET J.P., 1997. Élimination des pesticides par combinaison des procédés d'ozonation, d'adsorption sur CAP et d'ultra filtration. Deuxième Colloque International de Recherche sur les sous produits de traitement et d'épuration des fluides, Nuisances agricoles: Constats et solutions, GRUTTEE, Rennes (France), 19 et 20 mars 1997.

DUSART O., 1986. Modélisation des résultats de l'adsorption ultime de tensioactifs et de naphtol-2 sur charbon activé. Sci. de I'Eau, 5, 185-196.
GAID A., KAOUA F., MEDERRES N., 1994. Surface mass transfer processes using activated date pits as adsorbent, Water $S A, 20$ (4), 273-278.

GICQUEL L., WOLBERT D., LAPLANCHE A., 1997. Adsorption de l'atrazine par charbon actif en poudre : influence des matières organiques et minérales dissoutes des eaux naturelles. Environ. Technol., 18, 467-478.

LAFRANCE P., MAZET M., 1985. Adsorption des acides humiques sur charbon activé en poudre. Influence du tripolyphosphate de sodium. Wat. Res., 19, 1059-1064.

MANDRA V., ANSELME C., DANIEL L., 1993. Place de la nanofiltration dans une chaîne de traitement. l'Eau, l'Industrie et les Nuisances, 161, 54-58.

MAUPAS M., GILLET M., 1992. Premier bilan de la contamination de quelques rivières 
de Bretagne par les produits phytosanitaires. Colloque Phyt'eau, Versailles, 21-22 octobre.

MAZET M., YAACOUBI A., LAFRANCE P., 1988. The influence of metal ions released by an activated carbon on the adsorption of organics : the role of calcium ions. Wat. Res., 22 (10), 1321-1329.

PAILLARD H., CLERET D., BOURBIGOT M., 1990. Élimination des pesticides azotés par oxydation et adsorption sur charbon actif, Journées Intormation Eaux. $9^{e}$ Congrès, Poitiers, France.

PEREZ-CANDELA M., MARTIN-MARTINEZ J.M., TORREGROSA-MACI R., 1995. Chromium (VI) removal with activated carbon. Wat. Res., 29 (9), 2174-2180.

PRADOS M., CIBA N., 1997. Formation de sous-produits de latrazine dans les étapes d'oxydation et leur possible élimination sur charton actif, Deuxième Colloque International de Recherche sur les sous produits de traitement et d'épuration des fluides, Nuisances agricoles: Constat et solutions, GRUTTEE, Rennes (France), 19 et 20 mars 1997.
RANDTKE S.J., JEPSEN C., 1982. Effet of salts on activated carbon adsorption of fulvic acids. J. Am. Wat. Wks. Ass., 74, 84-93.

SELIM M.I., WANG J., 1994. Fate of atrazine in biologically active granular activated carbon. Environ. Toxicol. Chem., 13, 3-8.

SHARMA D.C., FORSTER C.F., 1996. Removal of hexavalent chronium from aqueous solutions by granular activated carbon. Water SA, 22 (2), 153-160.

SOUABI S., YAACOUBI A., MAZET M., 1994. Influence de l'ordre d'introduction des solutés (Naphtol-2 et Calcium) sur l'adsorption des tensioactifs sur charbon actif F400. Rev. Sci. Eau., 7, 43-54.

STUMM W., MORGAN J.J., 1981. "Aquatic chemistry $"$, Wiley inter-science, J.Wiley \& sons, $2^{\ominus}$ Ed.

WELTE B., MONTIEL A., DUPAS S., 1996. Evolution de la concentration en pesticides dans deux filières de traitement d'eau de surface. Source Resour., 3, 28-32.

YAACOUBI A., MAZET M., DUSART O., 1991. Compétition d'adsorption de deux solutés sur charbon actif. Wat. Res., 25 (8), 929-937. 